\title{
Remarks on the 3D Stokes eigenvalue problem under Navier boundary conditions
}

\author{
Alessio Falocchi ${ }^{1}$ (D) . Filippo Gazzola ${ }^{2}$
}

Received: 22 August 2021 / Accepted: 17 September 2021 / Published online: 9 October 2021

(c) The Author(s) 2021

\begin{abstract}
We study the Stokes eigenvalue problem under Navier boundary conditions in $C^{1,1}$-domains $\Omega \subset \mathbb{R}^{3}$. Differently from the Dirichlet boundary conditions, zero may be the least eigenvalue. We fully characterize the domains where this happens and we show that the ball is the unique domain where the zero eigenvalue is not simple, it has multiplicity three. We apply these results to show the validity/failure of a suitable Poincaré-type inequality. The proofs are obtained by combining analytic and geometric arguments.
\end{abstract}

Keywords Stokes eigenvalue problem $\cdot$ Navier boundary conditions

Mathematics subject classification $35 \mathrm{~K} 10 \cdot 47 \mathrm{~A} 75$

\section{Introduction}

Let $\Omega \subset \mathbb{R}^{3}$ be a bounded $C^{1,1}$-domain. The Stokes eigenvalue problem may be written as

$$
\begin{cases}-\Delta u+\nabla p=\lambda u & \text { in } \Omega, \\ \nabla \cdot u=0 & \text { in } \Omega,\end{cases}
$$

to be complemented with some boundary conditions; here, $\lambda \in \mathbb{R}$ is the eigenvalue, $u \in \mathbb{R}^{3}$ is the eigenvector (representing the velocity of a fluid in the context of Navier-Stokes equations), while $\nabla p$ stands for the gradient of the pressure $p$ of the fluid. In fact, (1) states that $u$ is an eigenvector, up to the addition of a gradient. This can be functionally characterized in the framework of the Helmholtz-Weyl decomposition [11, 21], see (5). So far, the Stokes eigenvalue problem (1) has been studied with precision only in special domains [8, 14-16]. All these works deal with Dirichlet boundary conditions and, only recently, the 2D problem under Navier boundary conditions was partially tackled in [3].

Alessio Falocchi

alessio.falocchi@polito.it

Filippo Gazzola

filippo.gazzola@polimi.it

1 Dipartimento di Scienze Matematiche, Politecnico di Torino, Torino, Italy

2 Dipartimento di Matematica, Politecnico di Milano, Milano, Italy 
Since $u \in \mathbb{R}^{3}$, also $\Delta u \in \mathbb{R}^{3}$. Moreover, $\nabla u$ is a $3 \times 3$ matrix. Denoting by

$$
\mathbf{D} u=\frac{\nabla u+\nabla^{\top} u}{2}
$$

the strain tensor, and exploiting the solenoidal property of $u$, one has that

$$
\Delta u=\operatorname{div} \nabla u=2 \operatorname{div} \mathbf{D} u
$$

and this "double" characterization of $\Delta u$ plays a fundamental role in the present paper. The most common boundary conditions complementing Eq. (1) are the homogeneous Dirichlet conditions

$$
u=0 \quad \text { on } \partial \Omega \text {, }
$$

also called no-slip boundary conditions. In 1827, Navier [12] proposed conditions with some friction, in which there is a stagnant layer of fluid close to the wall allowing a fluid to slip. In the zero-friction case, the homogeneous Navier boundary conditions read

$$
u \cdot v=(\mathbf{D} u \cdot v) \cdot \tau=0 \quad \text { on } \partial \Omega,
$$

where $v$ is the outward normal vector to $\partial \Omega$, while $\tau$ is tangential. The boundary conditions (4) turn out to be appropriate in many physically relevant cases [4, 9, 13]; see [6] for a survey of problems in which (4) arise. The first contribution to the Navier-Stokes equations under the Navier boundary conditions (4) is due to Solonnikov-Scadilov [18]; for subsequent results see [1, 2, 5-7].

The two boundary conditions (3) and (4) have in common that $u \cdot v=0$ on $\partial \Omega$ and their difference relies on the fact that the tangential request $u \cdot \tau=0$ on $\partial \Omega$ in (3) is replaced by $(\mathbf{D} u \cdot v) \cdot \tau=0$ on $\partial \Omega$ in (4). Once the boundary conditions are introduced, one needs to rigorously define weak solutions of (1). We are so led to recall the usual spaces in hydrodynamics

$$
\begin{gathered}
H=\left\{v \in L^{2}(\Omega) ; \nabla \cdot v=0, v \cdot v=0 \text { on } \partial \Omega\right\}, G=\left\{v \in L^{2}(\Omega) ; \exists g \in H^{1}(\Omega), v=\nabla g\right\}, \\
V=H \cap H^{1}(\Omega), \quad V_{0}=H \cap H_{0}^{1}(\Omega),
\end{gathered}
$$

in which the divergence has to be intended in weak form and where we denote by $v \cdot v$ the normal trace of $v$. Then $L^{2}(\Omega)=H \oplus G$ and $H \perp G$, where orthogonality is intended in $L^{2}(\Omega)$. It is well-known [10, Chapter III] that $H$ is a closed subspace of $L^{2}(\Omega)$; hence, $V$ and $V_{0}$ are closed subspaces of $H^{1}(\Omega)$. On these spaces we define the bilinear forms

$$
\begin{gathered}
(v, w)_{2}:=\int_{\Omega} v \cdot w \quad \forall v, w \in H, \\
(v, w)_{\mathbf{D}}:=\int_{\Omega} \mathbf{D} v: \mathbf{D} w, \quad(v, w)_{\nabla}:=\int_{\Omega} \nabla v: \nabla w \quad \forall v, w \in V .
\end{gathered}
$$

While the bilinear form $(\cdot, \cdot)_{2}$ defines a scalar product over $H$ (with corresponding norm $\|v\|_{2}^{2}=\int_{\Omega}|v|^{2}$ ), the situation is more delicate for the remaining two bilinear forms (here " : " denotes the scalar product between matrices). By using the solenoidal condition, it is straightforward that

$$
2(v, w)_{\mathbf{D}}=(v, w)_{\nabla} \quad \forall v, w \in V_{0} .
$$


The boundary conditions (3) or (4), associated with (1), strongly influence its weak formulation and the subsequent validity of Poincaré-type inequalities. Based on (2), and assuming the common condition $u \cdot v=0$ on $\partial \Omega$, in order to derive the weak formulation, there are two different ways of proceeding. The first way is to integrate by parts as in [6, Lemma 5.1] and to obtain

$$
-\int_{\Omega} \Delta u \phi=\int_{\Omega} \nabla u: \nabla \phi-\int_{\partial \Omega}(\operatorname{curl} u \wedge v) \cdot \phi+\int_{\partial \Omega} \phi \cdot \nabla v \cdot u \quad \forall u \in V \cap H^{2}(\Omega), \forall \phi \in V .
$$

The second way is to integrate by parts as in [2][(2.8)] and to obtain

$$
-\int_{\Omega} \Delta u \phi=2 \int_{\Omega} \mathbf{D} u: \mathbf{D} \phi \quad \forall u \in V \cap H^{2}(\Omega), \forall \phi \in V .
$$

By (2) and (7), the two identities (8) and (9) coincide in $V_{0}$ and simply become

$$
-\int_{\Omega} \Delta u \phi=\int_{\Omega} \nabla u: \nabla \phi \quad \forall u \in V_{0} \cap H^{2}(\Omega), \forall \phi \in V_{0} .
$$

Therefore, under the homogeneous Dirichlet boundary conditions (3) the weak formulation of (1)-(3) has a unique possible form which reads

$$
(u, \phi)_{\nabla}=\lambda(u, \phi)_{2} \quad \forall \phi \in V_{0} .
$$

But (8) and (9) have different consequences if set in the larger space $V$, namely if (1) is complemented with the Navier boundary conditions (4). In this case, we follow (9) and the weak form of (1)-(4) reads

$$
(u, \phi)_{\mathbf{D}}=\frac{\lambda}{2}(u, \phi)_{2} \quad \forall \phi \in V .
$$

By taking $\phi=u$, we see that the least eigenvalue $\lambda_{0}$ for both (11) and (12) is nonnegative: $\lambda_{0} \geq 0$.

Moreover, since $\lambda=0$ in (11) implies first that $\nabla u \equiv 0$ and then that $u \equiv 0$ in $\Omega$, it turns out that $\lambda_{0}>0$ in $V_{0}$; this allows to deduce that:

the Poincaré inequality $\lambda_{0}\|u\|_{2}^{2} \leq(u, u)_{\nabla}$ holds for some $\lambda_{0}>0$ and for any $u \in V_{0}$;

the norm $\|u\|_{\nabla}^{2}:=(u, u)_{\nabla}$ is equivalent to the $H^{1}(\Omega)$-norm in the space $V_{0}$.

On the other hand, by taking $\lambda=0$ in (12) one deduces that $\mathbf{D} u \equiv 0$ in $\Omega$ and one then wonders whether the same conclusions hold. This is why Amrouche-Rejaiba [2] introduce the kernel of the linear operator $u \mapsto \mathbf{D} u$ :

$$
\mathcal{T}:=\{u \in V ; \mathbf{D} u \equiv 0 \text { in } \Omega\} .
$$

If we restrict to the subspace $V_{0}$, this kernel is trivial: $\left\{u \in V_{0} ; \mathbf{D} u \equiv 0\right.$ in $\left.\Omega\right\} \equiv\{0\}$. But, if we merely require $u \cdot v=0$ on $\partial \Omega$ (as for the whole space $V$ ), the space $\mathcal{T}$ may be nontrivial. In this case, $V$ may be decomposed as direct sum of $\mathcal{T}$ and its orthogonal complement $\mathcal{T}^{\perp}$ :

$$
V=\mathcal{T} \oplus \mathcal{T}^{\perp}, \quad \forall v \in V \exists !\left(v_{\mathcal{T}}, v_{\mathcal{T}}^{\perp}\right) \in \mathcal{T} \times \mathcal{T}^{\perp} \quad \text { s.t. } \quad v=v_{\mathcal{T}}+v_{\mathcal{T}}^{\perp} .
$$

As we now state, the precise characterization of $\mathcal{T}$ depends on the geometry of $\Omega$. Our first purpose is to prove the following statement, complementing previous results in [2] and providing a different proof. 
Theorem 1 Let $\Omega \subset \mathbb{R}^{3}$ be a bounded $C^{1,1}$-domain. The kernel $\mathcal{T}$ has at most dimension 3 and, up to translations and rotations of the domain $\Omega$, it is spanned (at most) by the vectors

$$
W^{x}(y, z)=(0, z,-y), \quad W^{y}(x, z)=(-z, 0, x), \quad W^{z}(x, y)=(y,-x, 0) .
$$

The dimension of the kernel $\mathcal{T}$ depends on $\Omega$ and only three cases may occur:

$$
\operatorname{dim} \mathcal{T}=\left\{\begin{array}{l}
0 \text { if } \Omega \text { is not axisymmetric, } \\
1 \text { if } \Omega \text { is monoaxially symmetric, } \\
3 \text { if } \Omega \text { is a ball. }
\end{array}\right.
$$

Moreover, $\|\mathbf{D} \cdot\|_{2}$ and $\|\nabla \cdot\|_{2}$ are equivalent norms in $\mathcal{T}^{\perp}$ and there exists $C_{\Omega}>0$ such that

$$
\|v\|_{2} \leq C_{\Omega}\left\{\begin{array}{ll}
\|\mathbf{D} v\|_{2} & \text { if } \Omega \text { is not axisymmetric } \\
\left\|v_{\mathcal{T}}\right\|_{2}+\left\|\mathbf{D} v_{\mathcal{T}}^{\perp}\right\|_{2} & \text { if } \Omega \text { is axisymmetric }
\end{array} \quad \forall v \in V .\right.
$$

The first part of Theorem (1) means that the linearly independent vectors of the space $\mathcal{T}$ should be sought among $W^{x}, W^{y}, W^{z}$. By "equal left and right" we mean here that $\Omega$ has exactly one axis of (axial) symmetry.

Remark 1 The simplest examples of monoaxially symmetric domains are spheroids, namely ellipsoids having (only) two equal axes among the three. More examples of domains with $\mathcal{T} \neq\{0\}$ can be determined. Theorem 1 holds under the assumption that the domain is $C^{1,1}$ but, with little effort, this assumption can be relaxed: for instance, by requiring that $\partial \Omega \in C^{1,1}$ except for at most a set of null 2-dimensional Hausdorff measure. Hence, if $\Omega \subset \mathbb{R}^{3}$ is a circular cylinder (the Cartesian product between a planar disk and an interval), then $\operatorname{dim} \mathcal{T}=1$.

The second purpose of this paper is to connect Theorem 1 with the eigenvalue problem (1)-(4).

Corollary 1 Let $\Omega \subset \mathbb{R}^{3}$ be a bounded $C^{1,1}$-domain. Then one of the following facts holds:

if $\Omega$ is not axisymmetric, the least eigenvalue $\lambda_{0}$ of (1) is strictly positive: $\lambda_{0}>0$;

if $\Omega$ is monoaxially symmetric, then the least eigenvalue of (1) is $\lambda_{0}=0$ and is simple;

if $\Omega$ is a ball, then the least eigenvalue of (1) is $\lambda_{0}=0$ and has multiplicity 3.

A further consequence of Theorem 1 and Corollary 1 is that a Poincaré inequality such as $\lambda_{0}\|u\|_{2}^{2} \leq(u, u)_{\mathbf{D}}$ for some $\lambda_{0}>0$ and for all $u \in V$ holds if and only if $\Omega$ is not axisymmetric. Equivalently, as mentioned in [2, Lemma 3.3], the seminorm $u \mapsto(u, u)_{\mathbf{D}}^{1 / 2}$ is a norm over $V$ (equivalent to the $H^{1}$-norm) if and only if $\Omega$ is not axisymmetric.

As already mentioned, the least eigenvalue of (1)-(3) is strictly positive. Therefore, Corollary 1 is reminiscent of a similar phenomenon for the Laplacian of scalar functions: while under the Dirichlet boundary condition the least eigenvalue is strictly positive, under the Neumann boundary condition the least eigenvalue is zero and corresponds to constant eigenfunctions. For (1)-(4), the role of the constants is played by the kernel $\mathcal{T}$ that contains vectors $u$ such that $\mathbf{D} u \equiv 0$ : depending on the geometry of $\Omega$, this space may have dimensions 0,1 , or 3 .

The proofs of the above results are given in next section, they combine analytical and geometric arguments. Theorem 1 is proved in several steps. The first statement is obtained 
after differentiation of the PDE's characterizing the functions belonging to the kernel $\mathcal{T}$. The second statement is proved by solving a PDE that characterizes the geometric properties of the boundary $\partial \Omega$. The heart of the proof is (14): we show it by constructing an overdetermined boundary value problem for the torsion equation and by using a result by Serrin [17]. The remaining part of Theorem 1 and Corollary 1 take advantage of all these steps.

Although the focus of the present paper is the 3D case, let us conclude with a few words about different space dimensions. By using the simplest arguments in the proof of Theorem 1 , in the $2 \mathrm{D}$ case we can prove

Theorem 2 Let $\Omega \subset \mathbb{R}^{2}$ be a bounded $C^{1,1}$-domain. The kernel $\mathcal{T}$ is nontrivial if and only if $\Omega$ is a disk, in which case $\mathcal{T}$ has dimension 1 and is spanned by the vector $W(x, y)=(y,-x)$, up to constant vectors.

For larger space dimensions $n \geq 4$ the geometry becomes more complicated and this is why we leave the precise characterization of $\mathcal{T}$ as an open problem.

\section{Proofs}

The next geometric result is probably known. We provide here a purely analytical proof based on the celebrated work by Serrin [17].

Lemma 1 Let $\Omega \subset \mathbb{R}^{3}$ be an open and bounded $C^{1,1}$-domain, having two distinct axes of axial symmetry, then $\Omega$ is a ball.

Proof Consider the torsion problem

$$
\begin{cases}-\Delta g=1 & \text { in } \Omega, \\ g=0 & \text { on } \partial \Omega,\end{cases}
$$

that admits a unique solution, thereby having the same axial symmetries of $\Omega$. Let $r$ and $s$ be two distinct axes of axial symmetry for $\Omega$. If we compute $|\nabla g|$ in the points of $\partial \Omega$ lying on the same plane orthogonal to $r$, that we call meridians, we find $|\nabla g|=c_{1}$ for some $c_{1}>0$. Similarly, if we compute $|\nabla g|$ in the points of $\partial \Omega$ lying on the same plane orthogonal to $s$, that we call parallels, we find $|\nabla g|=c_{2}$ for some $c_{2}>0$. Since each parallel intersects infinitely many meridians and their union covers all $\partial \Omega$, we find $c_{1}=c_{2}=c$ and $|\nabla g|=c$ on $\partial \Omega$. Then (16) becomes an overdetermined problem with the further (Neumann) boundary condition

$$
\frac{\partial g}{\partial \nu}=-|\nabla g|=-c \quad \text { in } \Omega,
$$

where $v$ is the outward normal to $\partial \Omega$.

Since the overdetermined problem (16)-(17) does admit the solution $g$, by [17, Theorem 1] we infer that $\Omega$ is necessarily a ball if $\partial \Omega$ is assumed to be $C^{2}$; for merely $C^{1,1}$ boundaries, see the extension in the subsequent paper [20].

Proof of Theorem 1 Let $u:=\left(u^{1}, u^{2}, u^{3}\right) \in V$; the conditions $\mathbf{D} u \equiv 0$ in $\Omega$, characterizing the kernel $\mathcal{T}$, read 


$$
\begin{gathered}
u_{x}^{1}=u_{y}^{2}=u_{z}^{3}=0 \quad \text { in } \Omega \\
u_{y}^{1}+u_{x}^{2}=u_{z}^{2}+u_{y}^{3}=u_{z}^{1}+u_{x}^{3}=0 \quad \text { in } \Omega .
\end{gathered}
$$

From (18a) we deduce that $u^{1}=u^{1}(y, z), u^{2}=u^{2}(x, z)$ and $u^{3}=u^{3}(x, y)$ for all $(x, y, z) \in \Omega$. By formally differentiating the three Eq. in (18b) with respect to $x, y$ (first equation), $y, z$ (second equation), $x, z$ (third equation), and using (18a), we get

$$
\begin{array}{cccccccc}
u_{x y}^{1}+u_{x x}^{2}=0 & \Rightarrow & u_{x x}^{2}=0 & \text { and } & u_{y y}^{1}+u_{y x}^{2}=0 & \Rightarrow & u_{y y}^{1}=0 & \text { in } \Omega \\
u_{y z}^{2}+u_{y y}^{3}=0 & \Rightarrow & u_{y y}^{3}=0 & \text { and } & u_{z z}^{2}+u_{z y}^{3}=0 & \Rightarrow & u_{z z}^{2}=0 & \text { in } \Omega \\
u_{z z}^{1}+u_{z x}^{3}=0 & \Rightarrow & u_{z z}^{1}=0 & \text { and } & u_{x z}^{1}+u_{x x}^{3}=0 & \Rightarrow & u_{x x}^{3}=0 & \text { in } \Omega .
\end{array}
$$

Hence, there exist $a_{i}, b_{i}, c_{i}, d_{i} \in \mathbb{R}(i=1,2,3)$ such that

$$
\begin{array}{cc}
u^{1}(y, z)=a_{1}+b_{1} y+c_{1} z+d_{1} y z & \text { in } \Omega \\
u^{2}(x, z)=a_{2}+b_{2} x+c_{2} z+d_{2} x z & \text { in } \Omega \\
u^{3}(x, y)=a_{3}+b_{3} x+c_{3} y+d_{3} x y & \text { in } \Omega
\end{array}
$$

since $u$ is defined up to a gradient, we can take $a_{1}=a_{2}=a_{3}=0$. Through (18b) we then get

$$
\begin{aligned}
& b_{1}=-b_{2}, \quad d_{1}=-d_{2}, \quad c_{2}=-c_{3}, \quad d_{2}=-d_{3}, \quad c_{1}=-b_{3}, \quad d_{1}=-d_{3}, \\
& \Longrightarrow d_{1}=d_{2}=d_{3}=0 \text {. }
\end{aligned}
$$

Summarizing, we obtain that

$$
u(x, y, z)=\left(\begin{array}{c}
b_{1} y+c_{1} z \\
-b_{1} x+c_{2} z \\
-c_{1} x-c_{2} y
\end{array}\right) \in \operatorname{span}\left\{W^{x}(y, z), W^{y}(x, z), W^{z}(x, y)\right\}
$$

which shows that $u$ is smooth and justifies the above adopted formal procedure.

In fact, not necessarily all the three vectors $W$ are involved: they are solenoidal, but they also need to satisfy $W \cdot v=0$ on $\partial \Omega$ to ensure that $W \in V$. Let us focus on $W^{x}$, one can treat similarly $W^{y}$ and $W^{z}$. Let $v:=\left(v_{1}, v_{2}, v_{3}\right)$ be the unit outward normal to $\partial \Omega$ then

$$
W^{x} \cdot v=0 \text { on } \partial \Omega \quad \Longleftrightarrow \quad z v_{2}-y v_{3}=0 \quad \forall(x, y, z) \in \partial \Omega .
$$

Let $f:=f(x, y, z)$ the function defining $\partial \Omega$ through its zero level surface, that is,

$$
f(x, y, z)=0 \quad \Longleftrightarrow \quad(x, y, z) \in \partial \Omega .
$$

Assuming $\nabla f \neq 0$ on $\partial \Omega$, we have that $\nabla f$ is proportional to $v$. Therefore, (22) becomes $z f_{y}-y f_{z}=0$ on $\partial \Omega$, which shows that $f$ depends on $y$ and $z$ through $y^{2}+z^{2}$, hence $\Omega$ is axially symmetric with respect to the $x$-axis.

The above argument also shows that $\operatorname{dim} \mathcal{T}=0$ if $\Omega$ is not axis symmetric and, if $\operatorname{dim} \mathcal{T}>0$, then Lemma 1 applies and proves (14).

Finally, (15) follows from [19], see also [2, Lemma 3.3].

Proof of Corollary 1 It is a straightforward consequence of (14) in Theorem 1.

Proof of Theorem 2 Let $u:=\left(u^{1}, u^{2}\right) \in V$; the conditions $\mathbf{D} u \equiv 0$ in $\Omega$, characterizing the kernel $\mathcal{T}$, read 


$$
u_{x}^{1}=0, \quad u_{y}^{2}=0, \quad u_{y}^{1}+u_{x}^{2}=0, \quad \text { in } \Omega .
$$

The first two equations imply, respectively, that $u^{1}(x, y)=f(y)$ and $u^{2}(x, y)=g(y)$ for some functions $f$ and $g$. The third equation then implies that $f^{\prime}(y)+g^{\prime}(x)=0$, which is possible only if both $f^{\prime}$ and $g^{\prime}$ are constants. Hence, $f$ and $g$ are first order polynomials and, up to constant vectors, any $u \in \mathcal{T}$ is proportional to $W(x, y)=(y,-x)$.

By writing the boundary of $\partial \Omega$ in parametric form $(x=x(t), y=y(t))$ for $t$ in some compact interval $I$, and by imposing $W \cdot v=0$ on $\partial \Omega$, we infer that $W$ is proportional to the tangent vector $\left(x^{\prime}(t), y^{\prime}(t)\right)$, that is,

$$
x(t) x^{\prime}(t)+y(t) y^{\prime}(t)=\left|\begin{array}{ll}
y(t) & x^{\prime}(t) \\
-x(t) & y^{\prime}(t)
\end{array}\right| \equiv 0 \Longrightarrow x(t)^{2}+y(t)^{2}=R^{2}
$$

for some $R>0$, proving that, up to translations, $\Omega$ is a disk centered at the origin.

Acknowledgements The authors are supported by the PRIN project Direct and inverse problems for partial differential equations: theoretical aspects and applications and by INdAM.

Funding Open access funding provided by Politecnico di Torino within the CRUI-CARE Agreement.

Open Access This article is licensed under a Creative Commons Attribution 4.0 International License, which permits use, sharing, adaptation, distribution and reproduction in any medium or format, as long as you give appropriate credit to the original author(s) and the source, provide a link to the Creative Commons licence, and indicate if changes were made. The images or other third party material in this article are included in the article's Creative Commons licence, unless indicated otherwise in a credit line to the material. If material is not included in the article's Creative Commons licence and your intended use is not permitted by statutory regulation or exceeds the permitted use, you will need to obtain permission directly from the copyright holder. To view a copy of this licence, visit http://creativecommons.org/licenses/by/4.0/.

\section{References}

1. Acevedo, P., Amrouche, C., Conca, C., Ghosh, A.: Stokes and Navier-Stokes equations with Navier boundary condition. C.R. Math. Acad. Sci. Paris 357, 115-119 (2019)

2. Amrouche, C., Rejaiba, A.: $L^{p}$-theory for Stokes and Navier-Stokes equations with Navier boundary condition. J. Diff. Eq. 256, 1515-1547 (2014)

3. Arioli, G., Gazzola, F., Koch, H.: Uniqueness and bifurcation branches for planar steady Navier-Stokes equations under Navier boundary conditions. J. Math. Fluid. Mech. 23, 49 (2021)

4. Beavers, G.S., Joseph, D.D.: Boundary conditions at a naturally permeable wall. J. Fluid Mech. 30, 197-207 (1967)

5. Beirão da Veiga, H., : Regularity for Stokes and generalized Stokes systems under nonhomogeneous slip-type boundary conditions. Adv. Diff. Eq. 9, 1079-1114 (2004)

6. Berselli, L.C.: Some results on the Navier-Stokes equations with Navier boundary conditions. Riv. Math. Univ. Parma (N.S.) 1, 1-75 (2010)

7. Berselli, L.C.: An elementary approach to the 3D Navier-Stokes equations with Navier boundary conditions: existence and uniqueness of various classes of solutions in the flat boundary case. Discrete Contin. Dyn. Syst. Ser. S 3, 199-219 (2010)

8. Chen, L., Labrosse, G., Lallemand, P., Luo, L.S.: Spectrally accurate Stokes eigen-modes on isosceles triangles. Comput. Fluids 132, 1-9 (2016)

9. Galdi, G.P., Layton, W.J.: Approximation of the larger eddies in fluid motions. II. A model for spacefiltered flow. Math. Models Methods Appl. Sci. 10, 343-350 (2000)

10. Galdi, G.P.: An Introduction to the Mathematical Theory of the Navier-Stokes Equations: Steady-State Problems. Springer Science \& Business Media (2011) 
11. Helmholtz, H.: Über die theorie der elektrodynamic. Erste abhandlung. Über die bewegungsgleichungen der elektricität für ruhende leitende körper. J. Reine Ang. Math. 72, 57-129 (1870)

12. Navier, C.L.M.H.: Mémoire sur les lois du mouvement des fluides. Mem. Acad. Sci. Inst. Fr. 2, 389$440(1823)$

13. Pedlosky, J.: Geophysical Fluid Dynamics. Springer, New York (1979)

14. Rummler, B.: The eigenfunctions of the Stokes operator in special domains I. Z. Angew. Math. Mech. 77, 619-627 (1997)

15. Rummler, B.: The eigenfunctions of the Stokes operator in special domains II. Z. Angew. Math. Mech. 77, 669-675 (1997)

16. Rummler, B.: The eigenfunctions of the Stokes operator in special domains III. Z. Angew. Math. Mech. 82, 399-407 (2002)

17. Serrin, J.: A symmetry problem in potential theory. Arch. Rat. Mech. Anal. 43, 304-318 (1971)

18. Solonnikov, V.A., Scadilov, V.E.: A certain boundary value problem for the stationary system of Navier-Stokes equations. Tr. Mat. Inst. Steklova 125, 196-210 (1973)

19. Watanabe, J.: On incompressible viscous fluid flows with slip boundary conditions. J. Comput. Appl. Math. 159, 161-172 (2003)

20. Weinberger, H.F.: Remark on the preceding paper of Serrin. Arch. Rat. Mech. Anal. 43, 319-320 (1971)

21. Weyl, H.: The method of orthogonal projection in potential theory. Duke Math. J. 7, 411-444 (1940)

Publisher's Note Springer Nature remains neutral with regard to jurisdictional claims in published maps and institutional affiliations. 